\title{
Computational framework for the regularized 20-moment equations for non-equilibrium gas flows
}

\author{
S. Mizzi ${ }^{1,2}$, X. J. Gu ${ }^{1}$, D. R. Emerson ${ }^{1,2, *, \dagger}$, R. W. Barber ${ }^{1}$ and J. M. Reese ${ }^{2}$ \\ ${ }^{1}$ STFC Daresbury Laboratory, Keckwick Lane, Daresbury, Warrington WA4 4AD, U.K. \\ ${ }^{2}$ Department of Mechanical Engineering, University of Strathclyde, 75 Montrose Street, \\ Glasgow G1 1XJ, U.K.
}

\begin{abstract}
SUMMARY
This paper presents a framework for solving the regularized 20-moment equations consisting of a set of transport-like governing equations, the required constitutive closure, re-casting of the equations in second-order partial derivative form and derivation of additional wall boundary conditions. Couette flow results reveal that good agreement occurs between the 20-moment equations and direct simulation Monte Carlo data. Copyright (C) 2008 John Wiley \& Sons, Ltd.
\end{abstract}

Received 31 March 2007; Revised 4 December 2007; Accepted 5 December 2007

KEY WORDS: gas microfluidics; non-equilibrium gas flows; wall boundary conditions; method of moments

\section{INTRODUCTION}

The viable manufacture of miniaturized devices, on the order of microns or sub-microns, has been made possible through recent technological developments. However, these advances have not been matched by an improved fundamental understanding of the rich multiphysics occurring in micro-electro-mechanical systems (MEMS) [1]. In particular, microscale gas flows provide a lot of challenges in terms of predicting correctly and efficiently many of the observed nonequilibrium phenomena.

Non-equilibrium effects occur when the mean free path, $\lambda$, is similar to the characteristic length of the flow domain, e.g. channel height, $H$. The Knudsen number, $K n=\lambda / H$, is a dimensionless parameter characterizing non-equilibrium effects in gas flows and identifies the various

\footnotetext{
*Correspondence to: D. R. Emerson, Daresbury Laboratory, Keckwick Lane, Daresbury, Warrington WA4 4AD, U.K.

${ }^{\dagger}$ E-mail: d.r.emerson@dl.ac.uk

Contract/grant sponsor: Engineering and Physical Sciences Research Council; contract/grant number: GR/S77196/01
} 
regimes [2]. For $0.1<K n<10$, the flow is in the transition regime, and the Navier-Stokes-Fourier (NSF) equations are no longer considered to be valid due to the onset of various non-equilibrium effects. Alternative approaches are needed to model flows in this regime by means of either discrete methods or extended continuum modelling. Discrete methods such as direct simulation Monte Carlo (DSMC) provide a stochastic solution to the nonlinear Boltzmann equation but are known to be computationally intensive, especially for the low-speed flows typically encountered in MEMS [3].

The method of moments, developed by Grad [4], replaces the Boltzmann equation with a hierarchy of partial differential equations (PDEs) particularly providing closure for 13 equations (G13) where on top of the conservation equations of mass, momentum and energy additional balance laws for viscous stress and heat flux were derived. Torrilhon and Struchtrup [5] and Struchtrup [6,7] provided a different closure through regularization of Grad's equations (R13), which provide better resolution of non-equilibrium phenomena and are proven to be stable both in space and time. Gu and Emerson [8] cast the equations in a second-order PDE form and provide Maxwellian type wall boundary conditions (WBCs) [9]. Noticeable improvements over the classical NSF solutions were observed. This study follows a similar strategy using a regularized 20-moment (R20) equation set, which is derived together with the constitutive closure. Subsequently, the equations are re-cast in a second-order PDE form and additional WBCs are provided.

\section{R20 EQUATIONS}

The conservation equations for mass, momentum and energy in terms of position, $x_{i}$, and time, $t$, are given by

$$
\begin{gathered}
\frac{\mathrm{D} \rho}{\mathrm{D} t}+\rho \frac{\partial v_{i}}{\partial x_{i}}=0 \\
\rho \frac{\mathrm{D} v_{i}}{\mathrm{D} t}+\frac{\partial p}{\partial x_{i}}+\frac{\partial \tau_{i j}}{\partial x_{j}}=0
\end{gathered}
$$

and

$$
\frac{3}{2} \rho \frac{\mathrm{D} \theta}{\mathrm{D} t}+\frac{\partial q_{i}}{\partial x_{i}}+\left(p \delta_{i j}+\tau_{i j}\right) \frac{\partial v_{i}}{\partial x_{j}}=0
$$

where $\delta_{i j}$ is the Kronecker delta, $\rho$ is the density, $v_{i}$ is the flow velocity, $p=\rho \theta$ is the pressure and $\theta=R T$ is the specific energy related to the specific gas constant, $R$, and temperature, $T . \mathrm{D} / \mathrm{D} t$ is the material derivative and repeated indices indicate a tensor contraction. Relations for viscous stress, $\tau_{i j}$, and heat flux, $q_{i}$, are required in order to close the above five-moment equation set. The governing equations for $\tau_{i j}$ and $q_{i}$ derived by Grad [4] are expressed here together with an additional transport equation for $m_{i j k}$, where for Maxwell molecules,

$$
\frac{\mathrm{D} \tau_{i j}}{\mathrm{D} t}+\frac{\partial m_{i j k}}{\partial x_{k}}+\underline{2 p \frac{\partial v_{\langle i}}{\partial x_{j\rangle}}}+\frac{4}{5} \frac{\partial q_{\langle i}}{\partial x_{j\rangle}}+\tau_{i j} \frac{\partial v_{k}}{\partial x_{k}}+2 \tau_{k\langle i} \frac{\partial v_{j\rangle}}{\partial x_{k}}=-\frac{p \tau_{i j}}{\mu}
$$




$$
\begin{aligned}
\frac{\mathrm{D} q_{i}}{\mathrm{D} t}+ & \stackrel{\frac{1}{2} \frac{\partial R_{i j}}{\partial x_{j}}}{=}+\frac{1 \frac{\partial \Delta}{6} \frac{\partial \Delta}{\partial x_{i}}}{=}+m_{i j k} \frac{\partial v_{j}}{\partial x_{k}}+\frac{5 p}{2 \frac{\partial \theta}{\partial x_{i}}}+\theta \frac{\partial \tau_{i j}}{\partial x_{j}}+\frac{7}{2} \tau_{i j} \frac{\partial \theta}{\partial x_{j}}-\frac{\tau_{i j}}{\rho} \frac{\partial p}{\partial x_{j}}-\frac{\tau_{i j}}{\rho} \frac{\partial \tau_{j k}}{\partial x_{k}} \\
+\frac{7}{5} q_{i} \frac{\partial v_{j}}{\partial x_{j}}+\frac{7}{5} q_{j} \frac{\partial v_{i}}{\partial x_{j}} & +\frac{2}{5} q_{j} \frac{\partial v_{j}}{\partial x_{i}}=-\frac{2 p q_{i}}{3 \mu}
\end{aligned}
$$

and

$$
\begin{aligned}
& \frac{\mathrm{D} m_{i j k}}{\mathrm{D} t}+\underline{\underline{\underline{\partial x_{l}}}}+\underline{\underline{\frac{3}{7} \frac{\partial R_{\langle i j k}}{\partial x_{k\rangle}}}}+3 \theta \frac{\partial \tau_{\langle i j}}{\partial x_{k\rangle}}-\frac{3 \tau_{\langle i j}}{\rho}\left(\theta \frac{\partial \rho}{\partial x_{k\rangle}}+\frac{\partial \tau_{k\rangle l}}{\partial x_{l}}\right)+m_{i j k} \frac{\partial v_{l}}{\partial x_{l}} \\
& +3 m_{l\langle i j} \frac{\partial v_{k\rangle}}{\partial x_{l}}+\frac{12}{5} q_{\langle i} \frac{\partial v_{j}}{\partial x_{k\rangle}}=-\frac{3 p m_{i j k}}{2 \mu}
\end{aligned}
$$

where $R_{i j}, \Delta, m_{i j k}$ and $\phi_{i j k l}$ are higher-order moments and the angular brackets \langle\rangle represent the traceless part of a tensor. Regularization for the first five moments yields the NSF equations, as indicated by the single underlined terms in Equations (4) and (5). A similar regularization procedure on a 13-moment set would yield constitutive expressions similar to those derived by Torrilhon and Struchtrup [5], where

$$
\begin{gathered}
m_{i j k}{ }^{R 13}=-\frac{2 \mu}{p}\left(\theta \frac{\partial \tau_{\langle i j}}{\partial x_{k\rangle}}-\frac{\tau_{\langle i j}}{\rho}\left(\theta \frac{\partial \rho}{\partial x_{k\rangle}}+\frac{\partial \tau_{k\rangle l}}{\partial x_{l}}\right)+\frac{4}{5} q_{\langle i} \frac{\partial v_{j}}{\partial x_{k\rangle}}\right) \\
R_{i j}^{R 13}=-\frac{6 \mu}{7 p}\left(\frac{28}{5} \theta \frac{\partial q_{\langle i}}{\partial x_{j\rangle}}-\frac{8 \tau_{i j} \theta}{3} \frac{\partial v_{k}}{\partial x_{k}}+\frac{56}{5} q_{\langle i} \frac{\partial \theta}{\partial x_{j\rangle}}-\frac{28 q_{\langle i}}{5 \rho} \frac{\partial p}{\partial x_{j\rangle}}+4 \theta \tau_{k\langle i} \frac{\partial v_{j\rangle}}{\partial x_{k}}\right. \\
\left.+4 \theta \tau_{k\langle i} \frac{\partial v_{k}}{\partial x_{j\rangle}}-\frac{14 \tau_{i j}}{3 \rho} \frac{\partial q_{k}}{\partial x_{k}}-\frac{14 \tau_{i j} \tau_{k l}}{3 \rho} \frac{\partial v_{k}}{\partial x_{l}}-\frac{28 q_{\langle i}}{5 \rho} \frac{\partial \tau_{j\rangle k}}{\partial x_{k}}+\frac{2 p}{3 \mu \rho} \tau_{k\langle i} \tau_{j\rangle k}\right)
\end{gathered}
$$

and

$$
\Delta^{R 13}=-\frac{3 \mu}{2 p}\left(8 \theta \tau_{i j} \frac{\partial v_{i}}{\partial x_{j}}-\frac{8 q_{i}}{\rho} \frac{\partial p}{\partial x_{i}}-\frac{8 q_{i}}{\rho} \frac{\partial \tau_{i j}}{\partial x_{j}}+8 \theta \frac{\partial q_{i}}{\partial x_{i}}+28 q_{i} \frac{\partial \theta}{\partial x_{i}}+\frac{2 p \tau_{i j} \tau_{i j}}{3 \mu \rho}\right)
$$

From Equations (4)-(6), it can be shown that a closure relationship is required for $R_{i j}, \Delta$ and $\phi_{i j k l}$, indicated by the double-underlined terms. Equations (8) and (9) are used to provide closure relationships for $R_{i j}$ and $\Delta$, whereas $m_{i j k}$ will be solved in its full transport form requiring a closure for $\phi_{i j k l}$. The quantities $R_{i j}, m_{i j k}$ and $\phi_{i j k l}$ are traceless, dropping any angular brackets for clarity. Using a generic moment framework, regularization [7] and a production term for Maxwell molecules [10], it can be shown that [11]

$$
\begin{aligned}
\phi_{i j k l}= & \frac{2400 \mu}{5033 p}\left(-4 \theta \frac{\partial m_{\langle i j k}}{\partial x_{l\rangle}}+\frac{4 \theta}{\rho} m_{\langle i j k} \frac{\partial \rho}{\partial x_{l\rangle}}+\frac{4}{\rho} m_{\langle i j k} \frac{\partial \tau_{l\rangle m}}{\partial x_{m}}-12 \theta \tau_{\langle i j} \frac{\partial v_{k}}{\partial x_{l\rangle}}\right. \\
& \left.-\frac{233 p}{800 \mu \rho} \tau_{\langle i j} \tau_{k l\rangle}\right)
\end{aligned}
$$

In this paper, Equation (10) is used to close Equation (6). 


\section{NUMERICAL PROCEDURE}

Equations (1)-(5) can be re-cast in conservative form to yield similar expressions to those proposed by $\mathrm{Gu}$ and Emerson [8]. Using a similar procedure, Equation (6) is re-cast in conservative form where the convective, diffusive and source terms are identified by solving $m_{i j k}$ as a specific deviation from $m_{i j k}{ }^{R 13}$, where $\rho c_{i j k}=m_{i j k}-m_{i j k}{ }^{R 13}$. After some algebraic manipulation, with recursive use of tensor identities and substituting Equations (7) and $m_{i j k}=\rho c_{i j k}+m_{i j k}{ }^{R 13}$ in Equations (6) and (10), it can be shown that for steady state

$$
\begin{aligned}
& \underbrace{\frac{\partial \rho c_{i j k} v_{l}}{\partial x_{l}}}_{\text {convective term }}-\underbrace{\frac{\partial}{\partial x_{l}}\left(\frac{2400 \mu}{5033} \frac{\partial c_{i j k}}{\partial x_{l}}\right)}_{\text {diffusive term }} \\
& =\underbrace{-\frac{3 p \rho c_{i j k}}{2 \mu}-\frac{\partial}{\partial x_{l}}\left(m_{i j k}{ }^{R 13} v_{l}\right)-\frac{\partial E_{i j k l}}{\partial x_{l}}-\frac{3}{7} \frac{\partial R_{\langle i j}{ }^{R 13}}{\partial x_{k\rangle}}-3 m_{l\langle i j} \frac{\left.\partial v_{k}\right\rangle}{\partial x_{l}}}_{\text {source term }}
\end{aligned}
$$

where

$$
\begin{aligned}
E_{i j k l}= & \frac{2400 \mu}{5033 p}\left(-\theta c_{i j k} \frac{\partial \rho}{\partial x_{l}}-\theta \frac{\partial m_{i j k}^{R 13}}{\partial x_{l}}-\theta \frac{\partial m_{i j l}}{\partial x_{k}}-\theta \frac{\partial m_{i k l}}{\partial x_{j}}-\theta \frac{\partial m_{j k l}}{\partial x_{i}}+\frac{4 \theta}{\rho} m_{\langle i j k} \frac{\partial \rho}{\partial x_{l\rangle}}\right. \\
& \left.+\frac{4}{\rho} m_{\langle i j k} \frac{\partial \tau_{l\rangle m}}{\partial x_{m}}+\frac{12}{7} \theta \delta_{(i j} \frac{\partial m_{k l) m}}{\partial x_{m}}-12 \theta \tau_{\langle i j} \frac{\partial v_{k}}{\partial x_{l\rangle}}-\frac{233 p}{800 \mu \rho} \tau_{\langle i j} \tau_{k l\rangle}\right)
\end{aligned}
$$

Round brackets around subscripts $(i j k l)$ in Equation (12) indicate symmetrized tensors. In this form, additional WBCs for $c_{i j k}$ are required through arguments on $m_{i j k}$.

\section{MAXWELLIAN WBCs FOR $m_{i j k}$}

WBCs for $v_{i}, T, \tau_{i j}$ and $q_{i}$, suitable for a 13-moment set, were derived by Gu and Emerson [8] using a Grad 35-moment distribution function and a Maxwellian scattering kernel at the wall. The Maxwellian kernel is applicable to odd moments with respect to the normal velocity to the wall, $C_{2}$, so that the half flux integrals remain valid in the limit of a vanishing accommodation coefficient, $\alpha$ [4]. For a one-dimensional case, the number of independent variables is reduced to 12 and the moments considered at the wall are $\psi=\left(C_{2}, C_{2} C_{1}, C_{2} C^{2}, C_{2} C_{1} C_{1}, C_{2} C_{1} C_{2} C_{2}\right.$, $C_{2} C_{2} C_{2}, C_{2} C^{2} C_{1}, C_{2} C^{4}, C_{2} C_{1} C_{1} C_{1}, C_{2} C^{2} C_{1} C_{1}, C_{2} C_{1} C_{1} C_{1} C_{2} C_{2}, C_{2} C^{2} C_{2} C_{2}$ ), where $C_{1}$ is the peculiar velocity of molecules parallel to the wall. The boundary conditions are equivalent to those derived by $\mathrm{Gu}$ and Emerson [8] with the exception of $\tau_{22}$ and additional expressions for $m_{i j k}$, where

$$
\tau_{22}= \pm \kappa \frac{6 q_{2}+5 m_{222}}{15 \sqrt{\theta}}-\frac{2}{3}\left(\rho \theta-\sqrt{\frac{\theta_{w}}{\theta}} \rho_{w} \theta_{w}\right)-\frac{35 \phi_{2222}+30 R_{22}+7 \Delta}{420 \theta}
$$




$$
\begin{array}{r}
m_{111}= \pm \kappa \frac{21 \theta \tau_{12}+7 \phi_{1112}+3 R_{12}}{7 \sqrt{\theta}}-\frac{9 q_{1}}{5}-\frac{3}{2} m_{122}-\sqrt{\frac{\theta_{w}}{\theta}} v_{1 s} \rho_{w}\left(v_{1 s}^{2}+3 \theta_{w}\right) \\
m_{112}= \pm \frac{336 \theta\left(2 \tau_{11}+\tau_{22}\right)+420 \phi_{1122}-35 \phi_{2222}+9\left(16 R_{11}+8 R_{22}+7 \Delta\right)}{756 \kappa \sqrt{\theta}} \\
\pm \frac{1}{\kappa \sqrt{\theta}}\left(\frac{2}{3} \rho \theta^{2}-\frac{\rho_{w} v_{1 s}^{2}}{9} \sqrt{\frac{\theta_{w}}{\theta}}-\rho_{w} \theta_{w} \sqrt{\frac{\theta_{w}}{\theta}}\left(v_{1 s}^{2}+\frac{2 \theta_{w}}{3}\right)\right)-\frac{28 q_{2}}{45} \\
m_{122}= \pm \kappa \frac{7\left(3 \theta \tau_{12}+\phi_{1112}+\phi_{1222}\right)+6 R_{12}}{21 \sqrt{\theta}}-\frac{2}{3}\left(\frac{\rho_{w} \theta_{w}^{3 / 2} v_{1 s}\left(v_{1 s}^{2}+3 \theta_{w}\right)}{3 \theta^{3 / 2}}+q_{1}+\frac{m_{111}}{3}\right)
\end{array}
$$

and

$$
m_{222}= \pm \frac{336 \theta \tau_{22}+35 \phi_{2222}+72 R_{22}+21 \Delta}{126 \kappa \sqrt{\theta}} \pm\left(\frac{4 \rho \theta^{3 / 2}}{3 \kappa}-\frac{2 \rho_{w} \theta_{w}^{3 / 2}}{9 \kappa \theta}\left(v_{1 s}^{2}+6 \theta_{w}\right)\right)-\frac{28 q_{2}}{15}
$$

where $v_{1 s}$ is the slip velocity, $\kappa=\sqrt{\pi / 2}(2-\alpha) / \alpha$ and the subscript $w$ represents a wall condition yielding a density according to

$$
\rho_{w}=\frac{1}{\sqrt{\theta \theta_{w}}}\left(p+\frac{\tau_{22}}{2}-\frac{35 \phi_{2222}+7 \Delta+30 R_{22}}{840 \theta}\right)
$$

and a specific wall energy of $\theta_{w}=R T_{w}$.

\section{RESULTS}

Figure 1 compares Couette flow results for the proposed R20 equations against DSMC data and the results by $\mathrm{Gu}$ and Emerson [8] for $K n=0.25$ and a Mach number of 0.32. Argon was used as a model gas for a plate separation of $0.048 \mathrm{~m}$ at a pressure of $0.532 \mathrm{~Pa}$ with the wall temperatures set at $T_{w}=273 \mathrm{~K}$. No empirical corrections were imposed on the WBCs both on those derived by $\mathrm{Gu}$ and Emerson [8] and the additional conditions derived in this study.

\section{DISCUSSION AND CONCLUSIONS}

Figures 1(a), (c) and (e) show that the spurious behaviour close to the wall, reported for the R13 equations [8], is significantly attenuated with the R20 theory. Non-equilibrium effects are most significant in the Knudsen layer, a kinetic boundary layer occurring in close proximity to solid walls. This might indicate that larger moment sets improve the physical description of rarefied gas flows by making use of an extended approximation of the distribution function. The additional equations solved in combination with the newly derived boundary conditions are strongly coupled with the stress equation and contribute better to the diffusive process for stress, hence improving 

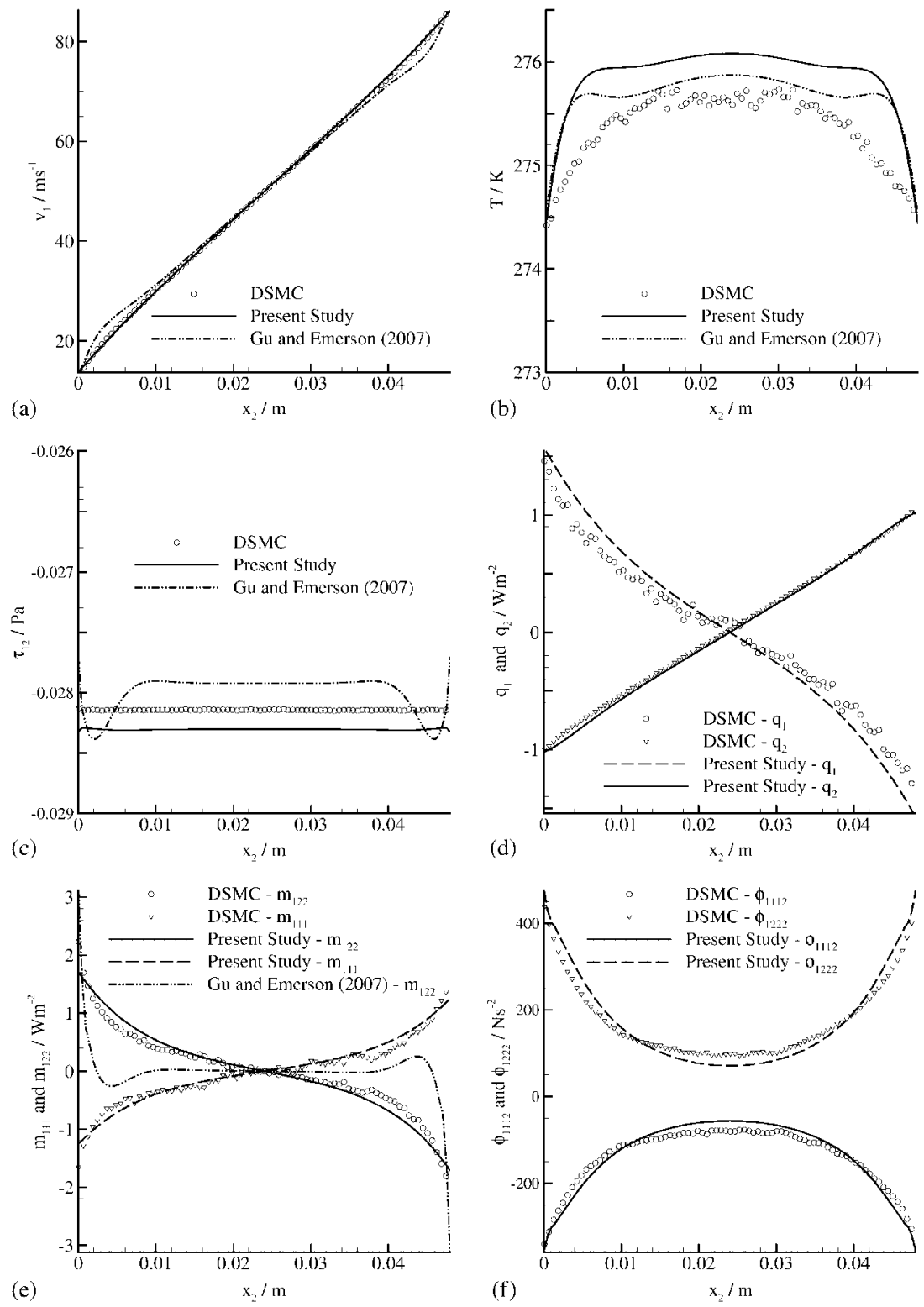

Figure 1. Couette flow results for $K n=0.25$; profiles for (a) tangential velocity, (b) temperature, (c) shear stress, (d) heat fluxes, (e) $m_{111}, m_{122}$ and (f) $\phi_{1112}, \phi_{1222}$.

the agreement with the DSMC data. However, from Figure 1(b), it is evident that the temperature profile is not so well resolved. It is unclear as yet whether this model artefact is a result of the constitutive theory or misrepresentation of the solid-wall treatment or a combination of both. From consideration of the constitutive theory, it can be seen that the closure relationships for the heat 
flux equation are common between the R13 and R20 theories. Resolution of these closure terms in their full transport form, by making use of a regularized 26-moment equation set, should improve the prediction of the temperature profile.

\section{REFERENCES}

1. Gad-el-Hak M. The MEMS Handbook. CRC Press: Boca Raton, FL, 2002.

2. Schaaf SA, Chambre PL. Flow of Rarefied Gases. Princeton University Press: Princeton, NJ, U.S.A., 1961.

3. Bird GA. Molecular Gas Dynamics and the Direct Simulation of Gas Flows. Oxford University Press: Oxford, U.K., 1994.

4. Grad H. On the kinetic theory of rarefied gases. Communications on Pure and Applied Mathematics 1949; 2:331-407.

5. Torrilhon M, Struchtrup H. Regularized 13 moment equations: shock structure calculations and comparison to Burnett models. Journal of Fluid Mechanics 2004; 513:171-198.

6. Struchtrup H. Stable transport equations for rarefied gases at high orders in the Knudsen number. Physics of Fluids 2004; 16:3921-3934.

7. Struchtrup H. Macroscopic Transport Equations for Rarefied Gas Flows. Springer: Berlin, 2005.

8. Gu XJ, Emerson DR. A computational strategy for the regularized 13 moment equations with enhanced wallboundary conditions. Journal of Computational Physics 2007; 225:263-283.

9. Maxwell JC. On stresses in rarefied gases arising from inequalities of temperature. Philosophical Transactions of the Royal Society (London) 1879; 170:231-256.

10. Truesdell C, Muncaster RG. Fundamentals of Maxwell's Kinetic Theory of a Simple Monatomic Gas. Academic Press: New York, 1980.

11. Mizzi S. Extended macroscopic models for rarefied gas dynamics in micro-sized domains. Ph.D. Thesis, to be submitted at the University of Strathclyde, 2008. 